\title{
TRANSLATING THE METHOD OF REDUCED MATRICES TO MACHINES I
}

\author{
Bernard A. Galler and Paul S. Dwyer \\ Department of Mathematics. \\ University of Michigan
}

\section{INTRODUCTION}

The method of reduced matrices was designed to provide an efficient method of solution for general transportation (or distribution) problems of the Hitchcock type [1] in which unit parcels at origins are to be transported to certain destinations, subject to a specified matrix of costs for moving one parcel from each origin to each destination, so as to minimize the total cost. Several other problems are mathematically equivalent to this transportation problem if one includes maximization problems as well as minimization problems. (Any maximization problem can be reduced to a corresponding minimization problem by multiplication of the elements of the cost matrix by -1.) Maximization problems include the assignment problem (which now has the connotation of assigning specific individuals to specific jobs), and the personnel classification problem in which individuals or groups of individuals are assigned in groups to jobs. Other equivalent mathematical problems are available in the literature.

Since the matrices of the grouped problems may be expanded to give square matrices with unit frequencies in each row and column, the essential mathematical problem may be interpreted as finding a permutation set of elements (one and only one element from each row and from each column) having optimal sum. In a grouped problem the grouping of this permutation set may be said to be a grouped per mutation set.

In the general transportation problem, as we use the term, we do not limit the discussion to two dimensions (as shown by the rows and columns of the specified matrix), but we consider a supermatrix (which we call a matrix) in $k$ dimensions which has the for $m$ of a mathematical cell having equal numbers off rows, columns, layers, etc. The objective is to find the permutation set of these elements (resulting from the selection of one and only one element from each row, column, layer, etc.) having optimal sum. As in the common case with $k=2$, grouping may frequently be applied to the permutation set as well as to the rows,

\footnotetext{
${ }^{1}$ This report was supported in whole or in part by the United States Air Force under Contract No. AF41(657)-9 monitored by the Operator Laboratory Field Unit No. 1, Air Force Personnel and Training Research Center, Lackland Air Force Base, Texas. This is not an official publication under the contract. Views or opinions expressed or implied herein a re not to be construed as necessarily reflecting the views or indorsement of the Department of the Air Force or of the Air Research and Development Command. Requests for additional copies should be addressed to: Engineering Research Institute, University of Michigan, Ann Arbor, Michigan.
} 
columns, layers, etc., but alternatively the matrix of each grouped problem may be interpreted as a larger matrix utilizing unit frequencies. In this sense the mathematical problem for this general case, as well as for the special case with $\mathrm{k}=2$, may be said to be the determination of a permutation set having optimal sum. Since maximization problems may be reduced directly to minimization problems as mentioned earlier, the rest of this discussion is limited to minimization problems.

This paper is intended to show how the method of reduced matrices has been translated into a workable computer program. Some of the details of the basic theory underlying this method have of necessity been omitted, and others have been modified in the process of adapting the method to machine computation. In particular, the transformation described in Section 6 (which is the most important feature of this method) is defined here in a way most suitable for automatic computation.

\section{THE OBJECTIVE OF THE METHOD OF REDUCED MATRICES}

The objective of the simplex method and other interchange methods as used in solving transportation-type problems with $\mathrm{k}=2$ is the deter mination of a feasible solution and the improvement of it by successive changes until the optimal solution is reached. The objective of this method of reduced matrices is to transform the matrix by operations which preserve the differences between the sums of the permutation sets. The resulting matrix consists of elements which are zero or positive, and in which some or all of the zero elements can be identified as those of a permutation set. Since the sum of the elements of this permutation set is zero, there can be no permutation set having a smaller sum. The objective of this method does not include the use of feasible solutions as does the simplex method, nor even the tentative partial assignment of frequencies to some of the elements presumably to be included in the solution as in Kuhn's method [11] for the assignment problem, or the Ford-Fulkerson* generalization of Kuhn's method for the transportation problem, but concentrates on the deter mination of transformations on the matrix which introduce additional zero terms. The aim here is to resolve the algebraic inconsistencies which appear in the application of the frequency conditions for each row, column, layer, etc. to the terms which constitute the trial permutation set. For many smaller problems to be worked by hand, especially with $k=2$, many of these inconsistencies can be determined readily by inspection and the whole reduction is accomplished informally.

An objective of the method, then, is to provide techniques which are especially designed to handle this class of problems (determination of permutation sets having optimal sums) and thus avoid the artificiality introduced when these problems are adjusted so as to utilize methods designed for more general problems. The artificiality of treating simpler cases as degenerate cases so that the formal requirements of the simplex method may hold, is a case in point. Also, the determination of a feasible solution (as has been pointed out by Schell [9]) becomes more difficult with increasing $\mathrm{k}$. These difficulties are avoided by directing the solution toward the basic mathematical problem, the determination of a permutation set having minimum sum.

The resulting solution is applicable to a wide class of problems in linear programming. An additional feature is that the machine process is the same for $k=2,3,4, \ldots$. No special

\footnotetext{
*Ford, L. R., Jr., and Fulkerson, D. R., "A simple algorithm for finding maximal network flows and an application to the Hitchcock problem," The Rand Corp., Paper P-743, December 29, 1955
} 
program is needed for the two-dimensional case, although additional techniques are available for two-dimensional problems to be worked less formally by hand.

In order to simplify the presentation, we are concentrating on a discussion of the case of $\mathrm{k}=3$. In general, the treatment of the $k=3$ case exhibits the chief difficulties (the elimination of negative solutions and the elimination of fractional solutions) of the general case. The $\mathrm{k}=2$ case is somewhat special in that there is no necessity for discussing the elimination of fractional solutions.

\section{THE GENERAL PROBLEM}

Let $c_{i j h}$ be the known cost of transporting a unit item at origin $i$ to destination $h$ through intermediate point $j$. Denote the number of parcels at $i$ by $f_{i}$, the number to be delivered to $h$ by $f_{h}$, and the capacity of the intermediate point by $f_{j}$. It is understood that the capacities of the intermediate points are adequate, i.e.,

$$
\sum_{i=1}^{n_{1}} f_{i}=\sum_{j=1}^{n_{2}} f_{j}=\sum_{h=1}^{n_{3}} f_{h}=N
$$

where $n_{1}, n_{2}$, and $n_{3}$ are the respective numbers of origins, intermediate points, and destinations. If we let $x_{i j h}$ be the number of units, zero or positive, which are assigned to route ijh, we wish to minimize the transportation cost

$$
T=\sum_{i, j, h} x_{i j h} c_{i j h}
$$

(summation is over the entire range of the subscript unless otherwise indicated). These values of $x_{i j h}$ must also satisfy the marginal conditions (which we call the equations)

$$
\sum_{j, h} x_{i j h}=f_{i}
$$

$$
\begin{aligned}
& \sum_{i, h} x_{i j h}=f_{j} \\
& \sum_{i, j} x_{i j h}=f_{h} .
\end{aligned}
$$

If $f_{i}=f_{j}=f_{h}=1$ with $n_{1}=n_{2}=n_{3}=N$, the values of $x_{i j h}$, as well as those of $c_{i j h}$, form an $N \times N \times N$ matrix. The set $S$ of triples $(i, j, h)$ with $x_{i j h}=1$, form a permutation set. Then (3.2) becomes

$$
\mathbf{T}=\sum_{\mathbf{S}} \mathbf{c}_{\mathrm{ijh}}
$$

so the problem is to find the permutation set $S$ whose associated $c_{i j h}$ have minimum sum.

Any problem with non-unit frequencies can be interpreted as a permutation set problem associated with an $N \times N \times N$ matrix. The grouped results of this expanded problem may 
be said to be a grouped permutation set consisting of the elements $(i, j, h)$ with the associated non-zero frequencies $x_{i j h}$. The problem is then the determination of integral $x_{i j h}>0$ which satisfy (3.3) and minimize (3.4). These values constitute a solution to the problem. The actual minimal sum may be obtained by substituting this grouped permutation set in (3.2).

\section{THE REDUCTION OF THE MATRIX}

Theoretically the type of transformation is simple. We subtract some constant from all elements of some particular row, column, or layer. Since in the ungrouped problem there must be one and only one selection from this row, column, or layer, the solution is not changed. Of course, the value of $T$ is changed by the amount subtracted. This concept can be immediately extended to grouped matrices since the grouped matrix may be rewritten as an $\mathbf{N} \times \mathbf{N} \times \mathbf{N}$ matrix with individual frequencies. Again the solution is not changed but the value of $\mathrm{T}$ is reduced by the total amount subtracted. Thus if $a_{i}$ is subtracted from each element of row $i$, which has frequency $f_{i}$, the value of $T$ is reduced by $f_{i} a_{i}$. This process may be applied simultaneously to as many rows, columns, and layers as desired.

\section{THE MARGINAL TRANSFORMATIONS}

The first steps in this method of reduced matrices utilize transfor mations which result in non-negative matrices with at least one zero in every row, column, and layer. This can be accomplished by subtracting consecutively the smallest element in each row from all the elements of that row, the smallest element of each column from all of the elements of that column, and similarly with each layer. The resulting matrix is said to be reduced.

In general there is nothing unique about a reduced matrix since the reductions in a $\mathrm{k}-$ dimensional problem can be performed in $\mathbf{k}$ ! different orders, and the resulting reduced matrix may be different for each order. When using hand methods, it seems better to compute the amount of the reduction of $T$ (the sum of the constants with frequencies taken into account) and to select the transformation having maximum reduction, i.e., first rows, then columns, then layers, or first columns, then layers, then rows, etc. For machine problems the subtractions in the order layers, columns, rows somewhat simplifies the process. After at most $\mathbf{k}$ steps, the matrix is reduced.

\section{THE GENERAL TRANSFORMATION}

Since this method of reduced matrices differs from other related methods most noticeably in the procedure used after the marginal transformations are completed, we are somewhat more formal in this section. Also, we state the problem in a slightly different way:

Given a set of equations

$$
A x=b
$$

where $A=\left(a_{i j}\right)$ is a $p \times q$ matrix with $p \leq q$ (here $p=n_{1}+n_{2}+\ldots+n_{k}$ and $\left.q=n_{1} n_{2} \ldots n_{k}\right)$, find that solution $x=\left(x_{1}, \ldots, x_{q}\right)$ in which each $x_{1}$ is a non-negative integer and such that

$$
T=\sum_{i=1}^{q} x_{1} c_{i}^{(0)}
$$


is minimized, where the $c_{i}{ }^{(0)}$ are bounded. (In the transportation problem at hand, we have $b \bullet 0$, and $a_{i j}=0$ or 1 [cf (3.3)].)

After the marginal transformations described in Section 5 , the numbers $c_{i}{ }^{(0)}$ have been changed into non-negative numbers $c_{j}$, with a reduction in $T$ of some amount $T_{0}$, i.e.,

$$
T-T_{0}=\sum_{i=1}^{q} x_{j} c_{i}^{(0)}-T_{0}=\sum_{i=1}^{q} x_{i} c_{i},
$$

and it is sufficient to minimize $T-T_{0}$. We assume that the $c_{i}$ are not all zero, otherwise any set of $q$ positive integers $x_{1}, \ldots, x_{q}$ constitutes a solution.

Let $\mathrm{Z}=\left\{\mathrm{j} \mid \mathrm{c}_{\mathrm{j}}=0\right\}$. We look for a solution of $(6.1)$ of the form $\mathrm{x}=\left(\mathrm{x}_{1}, \ldots, \mathrm{x}_{\mathrm{q}}\right) \geq 0$, $x_{j}=0$ whenever $j \notin Z$. (It is clear that for such a solution, $T-T_{0}$ is minimized.) Let $B$ be the matrix of coefficients of the system (6.1) subject to the condition $x_{j}=0$ for $j \notin Z$. In other words, B contains the $j$-th column of the matrix $A$ if and only if $c_{j}=0$. By a succession of row transformations, $B$ may be reduced to a matrix $D=\left(d_{i j}\right)$ (which is not necessarily unique) in echelon form, i.e., $D$ has the form

$$
D=\left(\begin{array}{ll}
I_{r} & D_{1} \\
0 & 0
\end{array}\right)
$$

where $r \leq p, I_{r}$ is the $r \times r$ identity matrix, and $D_{1}$ may be empty. Then $D=\pi B$, where $\pi=\left(\pi_{i j}\right)$ is the result of applying the same row transformations (in the same order) to the $p \times p$ identity matrix $I_{p}$. Let $b^{\prime}=\pi b$, i.e., $b_{i}^{\prime}=\sum_{j=1}^{p} \pi_{i j} b_{j}, i=1, \ldots, p$. Then for a solution of the kind we wish, we have

$$
\mathrm{Dx}=\pi \mathrm{Bx}=\pi \mathrm{b}=\mathrm{b}^{\prime}
$$

Suppose now that some row of $D$, say the $i_{0}$-th row, contains only zeros, while $b_{i_{0}}^{\prime} \neq 0$. (This implies that the $i_{0}$-th equation in the system (6.4) is not consistent with the other equations under our restriction on the for $m$ of the solution. Such a row is called an inconsistency row, and $b_{i}^{\prime}$ is called the amount of the inconsistency.) By performing additional row trans formations, if necessary, we may assume that for every inconsistency row $i, b_{i}^{\prime}>0$. We may also assume that among such rows, $b_{i_{0}}^{\prime}$ is maximal.

The existence of such inconsistency rows shows that a solution of the desired form is impossible unless the set $z$ is enlarged. In this case, we define $\theta$ by the equation

$$
\theta=\min _{\substack{\mathbf{j}_{\mathbf{i}_{0}, j} \mathbf{z}\\}}\left\{\frac{\mathbf{c}_{j}}{\mathbf{r}_{\mathbf{i}_{0}, j}}\right\}
$$


where $r_{i_{0}, j}=\sum_{k=1}^{p} \pi_{i_{0} k} a_{k j}, j=1, \ldots, q$. Such a number $\theta>0$ exists, since not all $c_{j}$ are zero, and if $r_{i_{0}, j} \leq 0$ for all $j$, we would have the contradiction (for any solution $\bar{x}=\left(\bar{x}_{1}, \ldots\right.$, $\left.\overline{\mathbf{x}}_{q}\right) \geq 0$ to $\left.(6.1)\right):$

$$
0 \geq \sum_{j=1}^{q} r_{i_{0}, j} \bar{x}_{j}=\sum_{j=1}^{q} \sum_{k=1}^{p} \pi_{1_{0} k} a_{k j} \bar{x}_{j}=\sum_{k=1}^{p} \pi_{i_{0} k} b_{k}=b_{i_{0}}^{\prime}>0 .
$$

It can be shown that $\theta$ is bounded from below by a number which depends only on the size of the original problem, since at each stage in the process the non-zero $c_{j}$ are bounded from below and the $\mathbf{r}_{\mathbf{i}_{0}, j}$ are bounded from above by such numbers.

We now define the transformation

$$
c_{j}^{\prime}=c_{j}-\theta r_{i_{0}, j}, \quad j=1,2, \ldots, q
$$

We observe that for $j \& Z$ we have $c_{j}^{\prime}=c_{j}=0$, since then $r_{i_{0}, j}=d_{i_{0} j}=0$ by our choice of $i_{0}$. It is clear, also, from (6.5) that for some $j_{1} \notin Z, \theta=\frac{c_{j_{1}}}{r_{i_{0}, j_{1}}}$, and we have $c_{j_{1}}^{\prime}=c_{j_{1}}-\theta r_{i_{0}, j_{1}}=$ $c_{j_{1}}-c_{j_{1}}=0$, so that $\mathrm{Z}$ is strictly increased to $\mathrm{Z}^{\prime}$ by the transformation. Moreover, since $c_{j} \geq 0, j=1,2, \ldots, q$, it follows that $c_{j}^{\prime} \geq 0, j=1,2, \ldots, q$. If, for some $j \varepsilon Z$, we had had $d_{i_{0} j}<0$ (cf Section 7 below) then $c_{j}^{\prime}=c_{j}-\theta r_{i_{0}, j}=-\theta d_{i_{0} j}>0$, although $c_{j}=0$, so that in this case $\mathbf{Z}^{\prime}$ would have fewer elements.

Since $b_{i_{0}}^{\prime}$ can also be shown to be bounded away from zero by a number depending only on the size of the original problem, it follows that $T^{\prime}=\sum_{j=1}^{q} \bar{x}_{j} c_{j}^{\prime}<T-T_{0}$ for any solution $\bar{x}=\left(\bar{x}_{1}, \ldots, \bar{x}_{q}\right)$ of $(6.1)$, since

$$
\begin{aligned}
T^{\prime} & =\sum_{j=1}^{q} c_{j}^{\prime} \bar{x}_{j}=\sum_{j=1}^{q}\left(c_{j}-\theta r_{i_{0}, j}\right) \bar{x}_{j}=\sum_{j=1}^{q} c_{j} \bar{x}_{j}-\theta \sum_{j=1}^{q} \sum_{k=1}^{p} \pi_{i_{0} k} a_{k j} \bar{x}_{j} \\
& =\left(T-T_{0}\right)-\theta \sum_{k=1}^{p} \pi_{i_{0} k} b_{k}=T-T_{0}-\theta b_{i}^{\prime}<T-T_{0} .
\end{aligned}
$$

Let $B^{\prime}$ be the matrix associated with the system (6.1) relative to $Z^{\prime}$ as $B$ was relative to $Z$. We may assume that the columns of $B^{\prime}$ consist of the columns of $B$ with any new columns adjoined at the right. The matrix $B^{\prime}$ may be reduced by row transformations to an echelon matrix $D^{\prime}$ of the form (6.3), thus generating a transformation matrix $\pi^{\prime}$, so that $D^{\prime}=\pi^{\prime} B^{\prime}$. We may also assume that in this reduction process those transfor mations which reduced $B$ to $D$ are applied first and in the same order. We shall show that $D^{\prime}$ has at least 
one less inconsistency row than $\mathrm{D}$, so that after a finite number of such transformations there will no longer be any inconsistency rows, i.e., the system of equations (6.1) will have a solution $\widetilde{x}=\left(\tilde{x}_{1}, \ldots, \widetilde{x}_{q}\right)$ such that $\tilde{x}_{j}=0$ whenever $c_{j}^{\prime} \neq 0$, where $c_{1}^{\prime}, \ldots, c_{q}^{\prime}$ are the transforms of $c_{1}, \ldots, c_{q^{*}}$ It is clear from our assumption on the generation of $\pi^{\prime}$ that all zero rows of $D^{\prime}$ are also zero rows of $D$, so that all inconsistency rows of $D^{\prime}$ are among those of $D$. We shall argue that the $i_{0}$-th row of $D^{\prime}$ is not an inconsistency row in $D^{\prime}$.

Suppose $D^{\prime}=\left(d_{i j}^{\prime}\right)$ has $d_{i_{0}}^{\prime}=0$ for all $j \in Z^{\prime}$. Then the elements of the $i_{o}$-th row of $\pi B^{\prime}$ can be expressed as linear combinations of the other rows of $\pi B^{\prime}, 1 . e$. , there are constants $q_{1}, \ldots, q_{s}$ such that $\sum_{k=1}^{p} \pi_{i_{0} k} a_{k j}=\sum_{i \neq i_{0}} \sum_{k=1}^{p} q_{i} \pi_{i k} a_{k j}$ for all $j \varepsilon z^{\prime}$. Let $\tilde{x}=$ $\left(\tilde{x}_{1}, \ldots, \tilde{x}_{q}\right)$ be any solution of the system of equations obtained by dropping the inconsistent equations from the system $B^{\prime} x=b^{\prime}$, so that, in particular, $\tilde{x}_{j}=0$ for $j \notin Z^{\prime}$. Then

$$
\begin{aligned}
\left(\pi^{\prime} b\right)_{i_{0}} & =b_{i_{0}}^{\prime}-\sum_{i \neq i_{0}} q_{i} b_{i}^{\prime}=\sum_{k=1}^{p} \pi_{i_{0} k} b_{k}-\sum_{i \neq i_{0}} \sum_{k=1}^{p} q_{i} \pi_{i k} b_{k} \\
& =\sum_{k=1}^{p}\left[\sum_{j=1}^{q} \pi_{i_{0}} a_{k j} \widetilde{x}_{j}-\sum_{k=1}^{p} \sum_{i \neq 1} \sum_{j=1}^{q} q_{i} \pi_{i k} a_{k j} \tilde{x}_{j}\right] \\
& =\sum_{j \varepsilon} z^{\prime}\left[\sum_{k=1}^{p} \pi_{i_{0} k} a_{k j}-\sum_{k=1}^{p} \sum_{i \neq i_{0}} q_{i} \pi_{i k} a_{k j}\right] \tilde{x}_{j} \\
& =0 \text {, so the } i_{o}-\text { th row of } D^{\prime} \text { is not an inconsistency row in } D^{\prime} .
\end{aligned}
$$

This process always transforms at least one inconsistency row to a consistency row at each step. (It may transform more than one.) We see then that a set of equations with $\mathrm{m}$ inconsistencies is reduced to a consistent set in at most $\mathrm{m}$ steps.

\section{ELIMINATION OF NEGATIVE SOLUTIONS}

When all inconsistency rows have been eliminated, a solution of the desired form can be found, and, in fact, is already at hand in the transform of the vector $b$ by the row transformations used in the reduction described in Section 6. Although this furnishes a solution to the system (6.1), it is an algebraic solution, in that the $x$ might be negative, and (except for $\mathbf{k}=2$ ) might be fractional.

Suppose, first, that the $i_{0}$-th row of $D$ contains at least one positive element and no negative elements, and $b_{i_{0}}^{\prime}<0$ (we use the same notation as in Section 6 , but we assume now that there are no inconsistency rows). By an additional row transformation, the $i_{0}$-th row may have all of its signs changed, so that now we would have $b_{i_{0}}^{\prime}>0$. We then perform the same transformation on the elements $c_{j}$ as in Section 6 , using the coefficients $\pi_{i_{o}}$ as before. It is easily verified that the following resultis are obtained: 
(1) For at least one $j_{1} \notin Z, \quad c_{j_{1}}^{\prime}=0$.

(2) If $d_{i_{0} j} \neq 0$ for some $j \in Z$, then $j \notin \mathbf{Z}^{\prime}$

(3) All $c_{j}^{\prime} \geq 0$ as before.

(4) $T-T_{0}$ is decreased as before by an amount $\theta b_{i_{0}}^{\prime}$ which is bounded from below.

Condition (4) guarantees that the process will stop after a finite number of steps, resulting in a positive (not necessarily integral) solution, if whenever $b_{j_{0}}^{\prime}<0$ (and the $i_{0}$-th row is not an inconsistency row), all the elements of the $i_{0}$-th row are non-negative.

\section{PERTURBATIONS}

One way of providing that the non-negativity condition of the previous section is satisfied is to arrange to have $D_{1}$ be empty in (6.3). To insure this it is sufficient to insure that $B$ have no more columns than rows, and this can be accomplished by insuring that all the $c_{j}(0)$ are distinct, so that there are exactly p-k zeros after the marginal transformations are completed, and also so that each transformation introduces exactly one new zero. Another way of arranging to have $D_{1}$ empty in (6.3) is to temporarily replace each $c_{j}=0$ whose associated column falls in $D_{1}$ by a small positive quantity $\varepsilon_{j}$. Once a solution is obtained to the resulting system, the columns of $D_{1}$ may be returned to the system, and the corresponding $x_{j}$ may play the role of parameters in the general solution.

The latter treatment is effectively carried out in hand computations, while the former is quite easily accomplished by a computer at the start of the computation. In order to make the $c_{j}{ }^{(0)}$ distinct, we perturb them by adding a (different) random number to each of them. These random numbers are added after first multiplying them by a sufficiently large negative power of two so that no accumulation of these numbers during the course of the computation can amount to as much as $1 / 2$ of the unit in which the $c_{j}$ are expressed. The probability that the random numbers used have the same sum for two permutation sets having the same optimal sum is extremely remote. If necessary, however, another set of random numbers may be used.

Such a procedure also modifies the problem so that it has a unique solution. By dropping these small random numbers we can then incorporate into the solution as parameters additional $x_{j}$ corresponding to the zeros thus obtained.

\section{THE ADJUSTMENT OF FRACTIONAL SOLUTIONS}

In a sense the attainment of a positive solution, fractional or integral, indicates the completion of the method of reduced matrices, even though an acceptable solution must be integral, since a matrix with a permutation set of zeros with fractional frequencies, subject only to the conditions stated earlier, can be reduced no further. This is shown by the fact that, if $t$ is a common denominator of all the fractions of the permutation set of zeros, the corresponding cost matrix is a completely reduced matrix for a related problem with identical $c_{j}$ values but with frequencies $t f$.

In a sense then we are faced with a second (combinatorial) problem. Using direct combinatorial methods we might select the elements corresponding to the zero terms of this matrix which is reduced as far as is possible, and then add in turn the terms corresponding to the smallest non-zero elements of this matrix and repeat the reduction process until a solution is reached. This technique is satisfactory for many practical problems since in many problems 
one of these small elements does complete the solution. However it is unsatisfactory theoretically, and it is easy to construct problems for which the proposal is obviously inefficient.

The method developed here uses the matrix $\pi$ resulting from the method of reduced matrices even though no further reductions of the matrix are possible. The elimination of fractional solutions by the addition to the solution of a single new element, as described below, has been incorporated into the program now in use on IBM 704 .

The method uses the particular fractional solution one gets by setting all parameters to zero in the solution obtained by the method of reduced matrices and modifies it into an integral solution with the addition of a new element which plays the role of a parameter. This means that the inclusion of the column associated with this element in the reduction of $B$ to $D$ leads to a reduced equation with zero in the column corresponding to the element, zero in the frequency column, and zero in the column corresponding to every element of the fractional solution. Furthermore, the coefficient of this element in the solution must be fractional and the fraction must be complementary to those of the particular fractional solution. These requirements give sets of necessary conditions which can be written in terms of the rows of $\pi$ and which must be satisfied if the element is to play the role of a parameter. Subsets of elements of the matrix are determined from these conditions, the element (or elements) to be adjoined to the solution set is then determined from these subsets, and the general solution is obtained.

Although the elimination of fractional solutions is an important feature of the method of reduced matrices, we have placed the emphasis in this paper on the determination of a positive solution, which may be fractional. Though improvements may be made in the method, we feel that the present techniques provide a practical and efficient method of solving this general transportation problem and that this method is generally superior, both by hand and by machine, to the simplex method even when $\mathrm{k}$ is as small as 2 .

\section{AN ILLUSTRATION}

To illustrate the method of reduced matrices, we consider a $\mathrm{k}=3,4 \times 3 \times 5$ problem proposed by Shell [9]. The matrix of cost elements $c_{i j h}$ is presented in Figure 1, with the associated row frequencies $f_{i}$, column frequencies $f_{j}$, and layer frequencies $f_{h}$ in the margins. Thus the element $c_{321}=34$ has row frequency 27 , column frequency 47 , and layer frequency 14.

\begin{tabular}{|c|c|c|c|c|c|c|c|c|c|c|c|c|c|c|c|}
\hline & \multicolumn{5}{|c|}{16} & \multicolumn{5}{|c|}{47} & \multicolumn{5}{|c|}{57} \\
\hline & 14 & 17 & 17 & 27 & 45 & 14 & 17 & 17 & 27 & 45 & 14 & 17 & 17 & 27 & 45 \\
\hline 44 & (2) & 3 & 43 & 47 & 14 & 44 & 47 & 2 & 23 & 19 & 18 & 2 & 46 & 42 & 23 \\
\hline 11 & 6 & 17 & 49 & 15 & 20 & 47 & 1 & 29 & 36 & 9 & 22 & 0 & 39 & 0 & 31 \\
\hline 27 & 17 & 36 & 47 & 30 & 21 & 34 & 31 & 18 & 2 & 14 & 47 & 44 & (1) & 40 & 33 \\
\hline 38 & 32 & 16 & 12 & 17 & 15 & 47 & 14 & 6 & 1 & 27 & 16 & 17 & 24 & 3 & \\
\hline
\end{tabular}

Figure 1 - Reduction in $T=2(14)+1(17)+1(45)=90$ 
Although the process to be described below is almost the same as that used in the computer program written for the IBM 704, the perturbation of the cost elements described in Section 8 above is omitted (there is a unique solution to this problem and the perturbation process is not needed) so the process can be seen more clearly.

\begin{tabular}{|c|c|c|c|c|c|c|c|c|c|c|c|c|c|c|c|}
\hline & \multicolumn{5}{|c|}{16} & \multicolumn{5}{|c|}{47} & \multicolumn{5}{|c|}{57} \\
\hline-2 & 14 & 17 & 17 & 27 & 45 & 14 & 17 & 17 & 27 & 45 & 14 & 17 & 17 & 27 & 45 \\
\hline 44 & 0 & 3 & 42 & 47 & 13 & 42 & 47 & (1) & 23 & 18 & 16 & 2 & 45 & 42 & 22 \\
\hline 11 & 4 & 17 & 48 & 15 & 19 & 45 & 1 & 28 & 36 & 8 & 20 & 0 & 38 & 0 & 30 \\
\hline 27 & 15 & 36 & 46 & 30 & 20 & 32 & 31 & 17 & 2 & 13 & 45 & 44 & 0 & 40 & 32 \\
\hline 38 & 30 & 16 & 11 & 17 & 14 & 45 & 14 & 5 & 1 & 26 & 14 & 17 & 23 & 3 & 0 \\
\hline
\end{tabular}

Figure $2-$ Reduction in $T=1(47)=47$

Figure 2 shows the result of subtracting the smallest element of each layer from each element of that layer (the elements transformed to zero are circled in each case). The numbers thus subtracted were 2, 0, 1, 0, 1, respectively, for a total reduction in $\mathrm{T}$ of 90 units (cf Section 4). A similar transformation performed on the three columns with frequency headings 16,47 and 57 yields a reduction in $\mathrm{T}$ of 47 , since the only subtraction needed to obtain a zero in each column is a subtraction of 1 from each element of the second column. At this point the matrix is reduced (cf Section 5), and row subtractions are unnecessary. The result of the column subtractions is shown in Figure 3.

\begin{tabular}{|c|c|c|c|c|c|c|c|c|c|c|c|c|c|c|c|}
\hline & \multicolumn{5}{|c|}{16} & \multicolumn{5}{|c|}{47} & \multicolumn{5}{|c|}{57} \\
\hline & 14 & 17 & 17 & 27 & 45 & 14 & 17 & 17 & 27 & 45 & 14 & 17 & 17 & 27 & 45 \\
\hline 44 & 0 & (3) & 42 & 47 & 13 & 41 & 46 & 0 & 22 & 17 & 16 & 2 & 45 & 42 & 22 \\
\hline 11 & 4 & 17 & 48 & 15 & 19 & 44 & 0 & 27 & 35 & 7 & 20 & 0 & 38 & 0 & 30 \\
\hline 27 & 15 & 36 & 46 & 30 & 20 & 31 & 30 & 16 & 1 & 12 & 45 & 44 & 0 & 40 & 32 \\
\hline 38 & 30 & 16 & 11 & 17 & 14 & 44 & 13 & 4 & 0 & 25 & 14 & 17 & 23 & 3 & 0 \\
\hline
\end{tabular}

Figure $3-$ Reduction in $\mathrm{T}=3(2)=6$

Now we pass to the more general type of transformation based on the elimination of inconsistent equations (cf Section 6). In Figure 4 the system $\mathrm{Bx}=\mathrm{b}$ is presented, with zeros suppressed, the location of the $c_{j}=0$ in the matrix at the head of each column, and the equations (3.4) identified at the left. (In the left-most column the first digit is the dimension and the second digit is the particular class within the dimension, so that the equation for Column 3 is listed as 23.) The column headed by 112 is the result of the transformation about to be described. 


\begin{tabular}{|l||ccccccccc|c||c|}
\hline & 111 & 123 & 222 & 232 & 234 & 333 & 424 & 435 & 112 & b \\
\hline 11 & 1 & 1 & & & & & & & 1 & 44 \\
12 & & & 1 & 1 & 1 & & & & & 11 \\
13 & & & & & & 1 & & & & 27 \\
14 & & & & & & & 1 & 1 & & 38 \\
\hline 21 & 1 & & & & & & & & 1 & 16 \\
22 & & 1 & 1 & & & & 1 & & & 47 \\
23 & & & & 1 & 1 & 1 & & 1 & & 57 \\
\hline 31 & 1 & & & & & & & & & 14 \\
32 & & & 1 & 1 & & & & & 1 & 17 \\
33 & & 1 & & & & 1 & & & & 17 \\
34 & & & & & 1 & & 1 & & & 27 \\
35 & & & & & & & & 1 & & 45 \\
\hline
\end{tabular}

Figure 4 - The system $B x=b$

It is not always necessary to reduce $B$ to the matrix $D$ in echelon for $m$ to find inconsistencies. It is obvious that $\mathrm{Eq}$. (21) and (31) cannot both be satisfied. (Since such obvious inconsistencies occur quite often, a search for the simple type that occurs here has been included in the 704 program.) In this case we can bypass the entire reduction process and go immediately into the transformation. The coefficients $\pi_{\mathbf{i}_{\mathbf{j}} \mathbf{j}}$ needed to define $\theta$ in this case are $(0,0,0,0,1,0,0,-1,0,0,0,0)$. (This corresponds to the eighth row of the $12 \times 12$ identity matrix after the transformations $31^{*}=31-21$ and $31^{* *}=-31^{*}$ are applied to it.) By means of (6.5) we find $\theta=\frac{c_{112}}{1}=3$ from Figure 3 , and the amount of the reduction in $T$ by this transformation is $3(2)=6$, since $b_{i_{0}^{\prime}}^{\prime}=2$ in this case. The result of this transformation (which causes the column headed 112 to be adjoined to $B$ in Figure 4) is shown in Figure 5.

\begin{tabular}{|c|c|c|c|c|c|c|c|c|c|c|c|c|c|c|c|}
\hline & \multicolumn{5}{|c|}{16} & \multicolumn{5}{|c|}{47} & \multicolumn{5}{|c|}{57} \\
\hline & 14 & 17 & 17 & 27 & 45 & 14 & 17 & 17 & 27 & 45 & 14 & 17 & 17 & 27 & 45 \\
\hline 44 & 0 & 0 & 39 & 44 & 10 & 44 & 46 & 0 & 22 & 17 & 19 & 2 & 45 & 42 & 22 \\
\hline 11 & 4 & 14 & 45 & 12 & 16 & 47 & 0 & 27 & 35 & 7 & 23 & 0 & 38 & 0 & 30 \\
\hline 27 & 15 & 33 & 43 & 27 & 17 & 34 & 30 & 16 & (1) & 12 & 48 & 44 & 0 & 40 & 32 \\
\hline 38 & 30 & 13 & 8 & 14 & 11 & 47 & 13 & 4 & 0 & 25 & 17 & 17 & 23 & 3 & 0 \\
\hline
\end{tabular}

Figure $5-$ Reduction in $T=1(38)=38$

Figure 6a shows the matrix D obtained at the next stage, except that the rows have not been rearranged to form an identity matrix as in (6.3). (Leaving the rows in their original 
order facilitates the machine computation somewhat.) It can be seen that there are two inconsistency rows, 33 and 35, with equal amounts of inconsistency. It does not matter which we choose to eliminate, and we simply choose the first, 33. The row of $\pi$ (cf Figure 6b) corresponding to this is also designated 33; from (6.5) we have $\theta=\frac{c_{324}}{1}=1$, and in the resulting transformation $\mathrm{T}$ is reduced by $1(38)=38$. The matrix one obtains at this stage is shown in Figure 7.

\begin{tabular}{|r||rrrrrrrrr||r|}
\hline & 111 & 123 & 222 & 232 & 234 & 333 & 424 & 435 & 112 & $b^{\prime}$ \\
\hline 11 & 1 & & & & & & & & & 14 \\
12 & & & 1 & & & & & & & -12 \\
13 & & & & & & 1 & & & & 27 \\
14 & & & & & & & 1 & & & 31 \\
\hline 21 & & 1 & & & & & & & & 28 \\
22 & & & & 1 & & & & & & 27 \\
23 & & & & & & & & & & \\
\hline 31 & & & & & & & & & 1 & 2 \\
32 & & & & & 1 & & & & & -4 \\
33 & & & & & & & & & & 38 \\
34 & & & & & & & & 1 & & 7 \\
35 & & & & & & & & & & 38 \\
\hline
\end{tabular}

Figure 6a - The matrix D

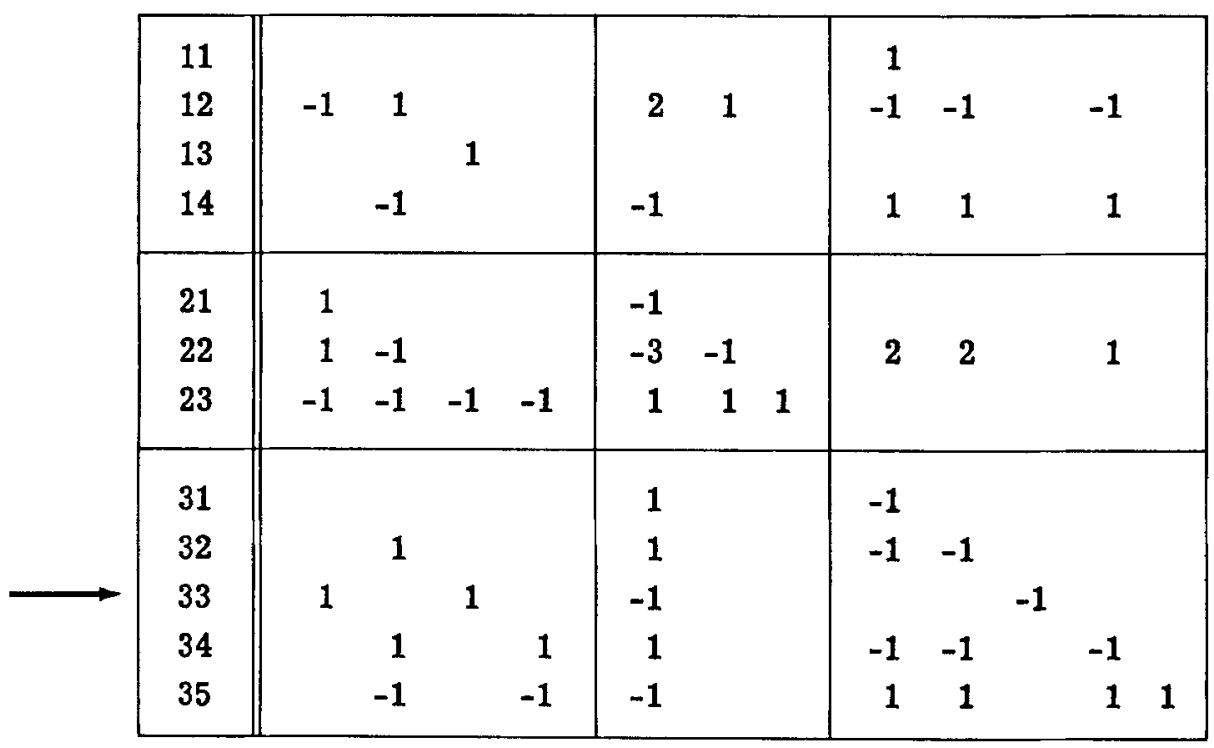

Figure $6 b-$ The matrix $\pi$ 


\begin{tabular}{|c|c|c|c|c|c|c|c|c|c|c|c|c|c|c|c|}
\hline & \multicolumn{5}{|c|}{16} & \multicolumn{5}{|c|}{47} & \multicolumn{5}{|c|}{57} \\
\hline & 14 & 17 & 17 & 27 & 45 & 14 & 17 & 17 & 27 & 45 & 14 & 17 & 17 & 27 & 45 \\
\hline 44 & 0 & 0 & 40 & 44 & 10 & 43 & 45 & 0 & 21 & 16 & 18 & & 45 & 41 & 21 \\
\hline 11 & 5 & 15 & 47 & 13 & 17 & 47 & 0 & 28 & 35 & 7 & 23 & 0 & 39 & 0 & 30 \\
\hline 27 & 15 & 33 & 44 & 27 & 17 & 33 & 29 & 16 & 0 & 11 & 47 & 43 & 0 & 39 & 31 \\
\hline 38 & 31 & 14 & 10 & 15 & 12 & 47 & 13 & 5 & 0 & 25 & 17 & 17 & 24 & 3 & 0 \\
\hline
\end{tabular}

Figure $7-$ Reduction in $T=\frac{1}{2}(12)=6$

The process is repeated in Figure 8a, but this time there are no inconsistencies. (Note that two inconsistencies were eliminated with one transformation.) We have an algebraic solution in the column headed $b^{\prime}$, but there are four negative values. We use the largest (in size) to generate a transformation (cf Section 7). We apply one more row transformation, $12^{*}=\mathbf{- 1 2}$, to the matrix $\pi$ shown in Figure $8 \mathrm{~b}$, and the second row, used to find $\theta$, is then

$$
(1,-1,0,0,-2,-1,0,1,1,0,1,0) \text {. }
$$

This time $\theta=\frac{c_{132}}{2}=\frac{1}{2}$, and the reduction in $T$ is $\frac{1}{2}(12)=6$. The result is shown in Figure 9 .

\begin{tabular}{|r||rrrrrrrrrr||r|}
\hline & 111 & 123 & 222 & 232 & 234 & 333 & 424 & 435 & 112 & 324 & $b^{\prime}$ \\
\hline 11 & 1 & & & & & & & & & & 14 \\
12 & & & 1 & & & & & & & & -12 \\
13 & & & & & & 1 & & & & & -11 \\
14 & & & & & & & 1 & & & & -7 \\
\hline 21 & & 1 & & & & & & & & & 28 \\
22 & & & & 1 & & & & & & & 27 \\
23 & & & & & & & & & & & \\
\hline 31 & & & & & & & & & 1 & & 2 \\
32 & & & & & 1 & & & & & & -4 \\
33 & & & & & & & & & & 1 & 38 \\
34 & & & & & & & & 1 & & & 45 \\
35 & & & & & & & & & & & \\
\hline
\end{tabular}

Figure $8 \mathrm{a}$ - The matrix $\mathrm{D}$ 


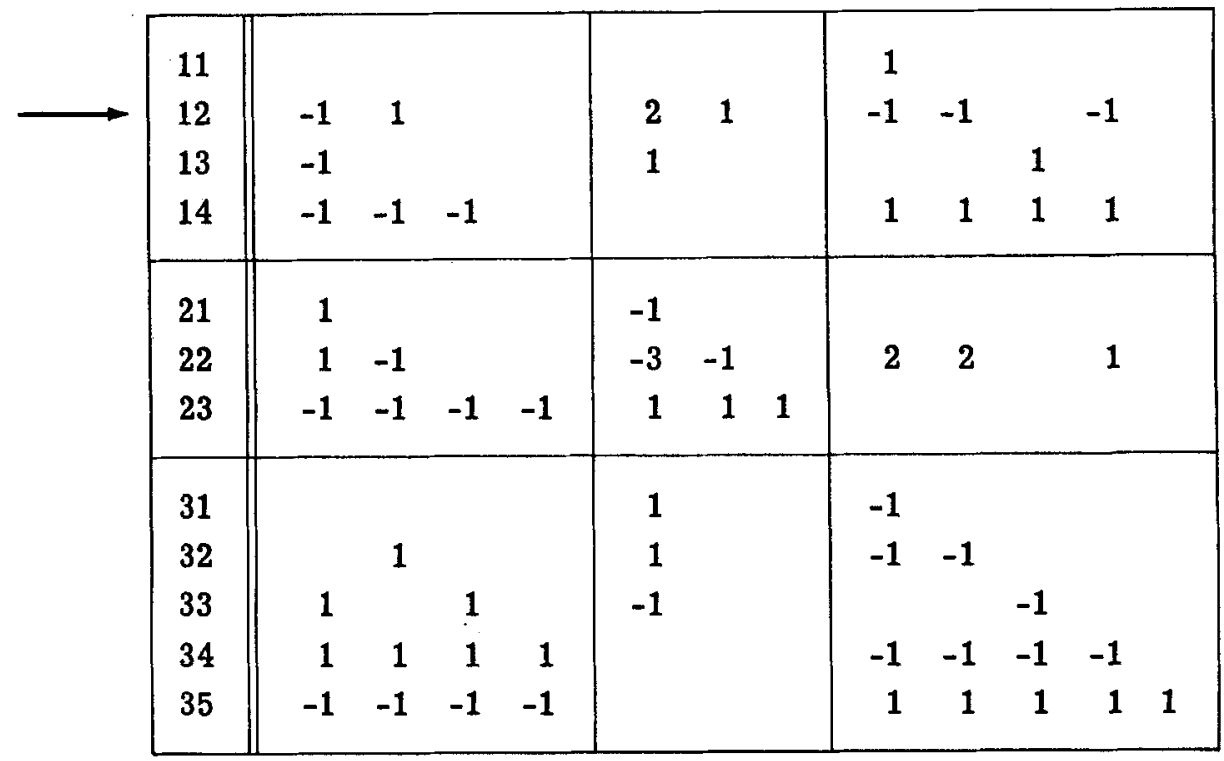

Figure $8 \mathrm{~b}$ - The matrix $\pi$

\begin{tabular}{|c|c|c|c|c|c|c|c|c|c|c|c|c|c|c|c|}
\hline & \multicolumn{5}{|c|}{16} & \multicolumn{5}{|c|}{47} & \multicolumn{5}{|c|}{57} \\
\hline $\mathrm{f}_{\mathrm{h}}$ & 14 & 17 & 17 & 27 & 45 & 14 & 17 & 17 & 27 & 45 & 14 & 17 & 17 & 27 & 45 \\
\hline 44 & 0 & 0 & 40.5 & 44 & 10.5 & 42.5 & 44.5 & 0 & 20.5 & 16 & 17 & 0 & 44.5 & 40 & 20.5 \\
\hline 11 & 6 & 16 & 48.5 & 14 & 18.5 & 47.5 & .5 & 29 & 35.5 & (8) & 23 & 0 & 39.5 & 0 & 30.5 \\
\hline 27 & 15.5 & 33.5 & 45 & 27.5 & 18 & 33 & 29 & 16.5 & 0 & 11.5 & 46.5 & 42.5 & 0 & 38.5 & 31 \\
\hline 38 & 31.5 & 14.5 & 11 & 15.5 & 13 & 47 & 13 & 5.5 & 0 & 25.5 & 16.5 & 16.5 & 24 & 2.5 & 0 \\
\hline
\end{tabular}

Figure $9-$ Reduction in $\mathrm{T}=8(7)=56$

Note that $c_{222}$ (which gave the solution value -12 ) is no longer zero after this transformation. It has been replaced by $c_{132}$. We observe also that the number of negative solution values has been reduced by two.

The process is repeated, as is shown in Figures $10 \mathrm{a}$ and $10 \mathrm{~b}$, with $\theta=\frac{\mathrm{c}_{225}}{1}=8$, and $\mathrm{T}$ reduced by $8(7)=56$. The result is shown in Figure 11 .

\begin{tabular}{|c|c|c|c|c|c|c|c|c|c|c|c|}
\hline & 111 & 123 & 232 & 234 & 333 & 424 & 435 & 112 & 324 & 132 & $\mathbf{b}^{\prime}$ \\
\hline $\begin{array}{l}11 \\
12 \\
13 \\
14\end{array}$ & 1 & & & & 1 & 1 & & & & 1 & $\begin{array}{r}14 \\
6 \\
-5 \\
-7\end{array}$ \\
\hline $\begin{array}{l}21 \\
22 \\
23\end{array}$ & & 1 & 1 & & & & & & & & $\begin{array}{r}22 \\
9\end{array}$ \\
\hline $\begin{array}{l}31 \\
32 \\
33 \\
34 \\
35\end{array}$ & & & & 1 & & & 1 & 1 & 1 & & $\begin{array}{r}2 \\
2 \\
32 \\
45\end{array}$ \\
\hline
\end{tabular}

Figure 10a - The matrix D 
TRANSLATING METHOD OF REDUCED MATRICES TO MACHINES

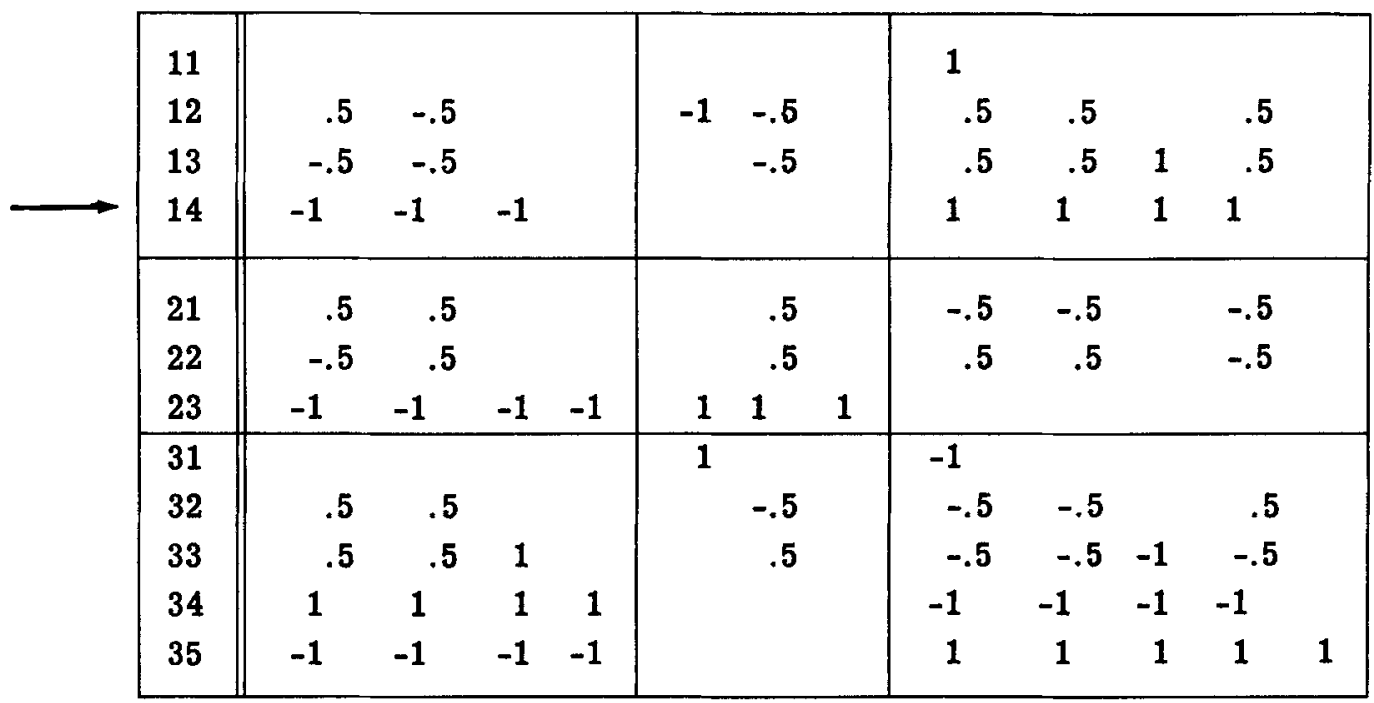

Figure $10 \mathrm{~b}$ - The matrix $\pi$

\begin{tabular}{|c|c|c|c|c|c|c|c|c|c|c|c|c|c|c|c|}
\hline $\mathbf{f}_{\mathbf{j}}$ & \multicolumn{5}{|c|}{16} & \multicolumn{5}{|c|}{47} & \multicolumn{5}{|c|}{57} \\
\hline & 14 & 17 & 17 & 27 & 45 & 14 & 17 & 17 & 27 & 45 & 14 & 17 & 17 & 27 & 45 \\
\hline 44 & 0 & 0 & 40.5 & 44 & 2.5 & 42.5 & 44.5 & 0 & 20.5 & 8 & 17 & 0 & 44.5 & 40 & 12.5 \\
\hline 11 & 6 & 16 & 48.5 & 14 & 10.5 & 47.5 & .5 & 29 & 35.5 & 0 & 23 & 0 & 39.5 & 0 & 22.5 \\
\hline 27 & 15.5 & 33.5 & 45 & 27.5 & 10 & 33 & 29 & 16.5 & 0 & 3.5 & 46.5 & 42.5 & 0 & 38.5 & 23 \\
\hline 38 & 39.5 & 22.5 & 19 & 23.5 & 13 & 55 & 21 & 13.5 & 8 & 25.5 & 24.5 & 24.5 & 32 & 10.5 & 0 \\
\hline
\end{tabular}

Figure 11 - Finally reduced matrix

At this point the solution has been achieved, as is seen in Figure 12a, where $b^{\prime}$ contains only non-negative integers, and all equations are consistent.

\begin{tabular}{|r||rrrrrrrrrr||r|}
\hline & 111 & 123 & 232 & 234 & 333 & 435 & 112 & 324 & 132 & 225 & $b^{\prime}$ \\
\hline 11 & 1 & & & & & & & & & & 14 \\
12 & & & & & & & & & 1 & & 13 \\
13 & & & & & 1 & & & & & & 2 \\
14 & & & & & & & & & & 1 & 7 \\
\hline 21 & & 1 & & & & & & & & & 15 \\
22 & & & 1 & & & & & & & & 2 \\
23 & & & & & & & & & & & \\
\hline 31 & & & & & & & 1 & & & & 2 \\
32 & & & & 1 & & & & & & & 2 \\
33 & & & & & & & & 1 & & & 25 \\
34 & & & & & & 1 & & & & & 38 \\
35 & & & & & & & & & & & \\
\hline
\end{tabular}




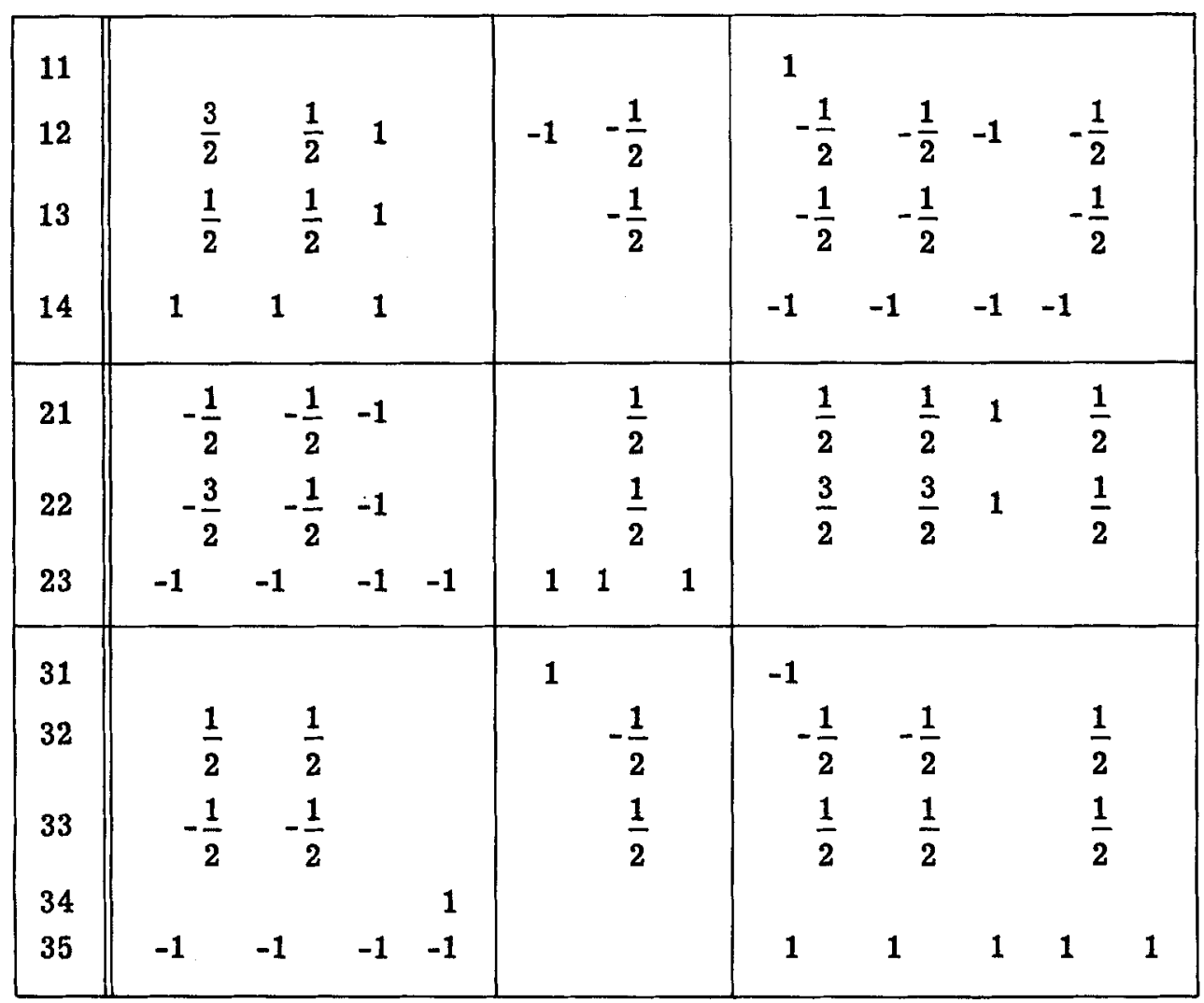

Figure $12 \mathrm{~b}$ - The matrix $\pi$

Figure 13 shows the solution values in the corresponding locations in the matrix (with zeros suppressed). The minimal value of $T$ may now be computed in either of two ways. One may total up the successive reductions in $T$ resulting from the transformations performed:

$$
90+47+6+38+6+56=243 \text { units }
$$

or one may apply (3.2) directly:

$14(2)+15(2)+2(0)+2(0)+2(1)+38(1)+2(3)+25(2)+13(2)+7(9)=243$ units

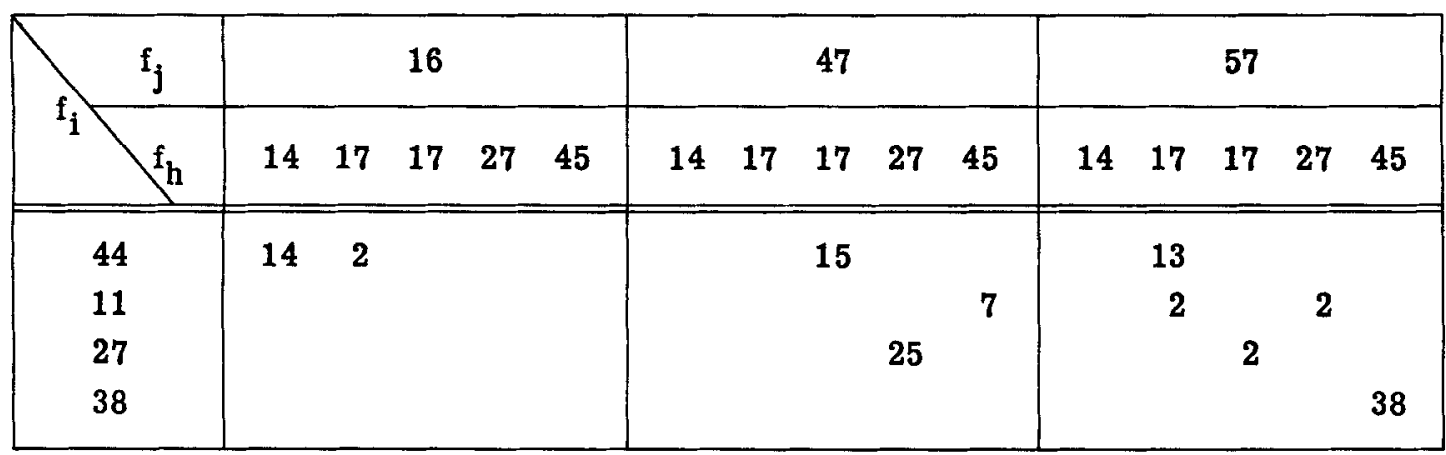

Figure 13 - The solution

The computation time for this problem on the IBM 704 was 1.3 minutes. 


\section{REFERENCES}

A. Problems with $\mathrm{k}=\mathbf{2}$

[1] Hitchcock, Frank L., "The distribution of a product from several sources," Math. Phys 20, 224-230 (1941)

[2] Dantzig, George B., "Application of the simplex method to a transportation problem," Chapter XXIII, Activity analysis of production and allocation, Cowles Commission Monograph No. 13, New York:Wiley, (1951)

[3] Votaw, D. F., Jr., "Methods of solving some personnel-classification problems," Psychometrika, 17, 255-266 (1952)

[4] Flood, Merrill M., "On the Hitchcock distribution problem," Pac. J. Math., 3, 369-386 (1953)

[5] von Neumann, J., "A certain zero-sum two-person game equivalent to the optimal assignment problem," Contributions to the Theory of Games II, edited by $\mathrm{H}$. W. Kuhn and A. W. Tucker, Annals of Mathematics Studies 28, 5-12, Princ eton (1953)

[6] Dwyer, P. S., "Solution of the personnel classification problem with the method of optimal regions," Psychometrika, 19, 11-26 (1954)

B. Problems with $\mathrm{k}>2$

[7] Motzkin, T. S., "The multi-index transportation problem," Bull. Am. Math. Soc. 58,494 (1952)

[8] Roby, T. B., "Problems of rational group assembly exemplified in the medium bomber crew," Research Bulletin 53-18, Human Resources Research Center, Lackland Air Force Base, San Antonio, July 1953

[9] Schell, Emil D., "Distribution of a product of several properties," presented to Linear Programming Symposium, National Bureau of Standards, Washington, Jan. 29, 1955

[10] Dwyer, Paul S., "Development of generalized mathematical procedures for optimal assembly of potentially effective bomber crews," Research Bulletin to be published by Human Resources Research Center, Lackland Air Force Base, San Antonio

C. Methods of reduced matrices

[11] Kuhn, H. W., "A combinatorial algorithm for the assignment problem," Issue 11 of Logistics Papers, George Washington University Logistics Research Project, 1955. See also "The Hungarian Method for the assignment problem," Naval Research Logistics Quarterly, Vol. 2, March-June, 1955

[12] Flood, Merrill M., "The travelling salesman problem," J. Opns. Res. Soc. Am. 4, 61-75 (1956)

[13] Dwyer, Paul S. and Galler, Bernard A., "The method of reduced matrices for a general transportation problem," presented to the Los Angeles meeting of the Association for Computing Machinery, August, 1956 